\title{
Electron Microscopy Studies of Martensite Microstructures
}

\author{
D. Schryvers \\ EMAT, University of Antwerp, RUCA, Groenenborgerlaan 171, 2020 Antwerp, Belgium
}

\begin{abstract}
An overview will be given of some recent results of microstructural electron microscopy studies on martensitic systems. The precursor structure observed in a ternary Ni-Mn-Ti alloy and its correlation with the ensuing stacking sequence is presented. New types of materials of a well known alloy, in casu splat-cooled and nanoscale thin films of $\mathrm{Ni}-\mathrm{Al}$ are investigated revealing particular morphologies of the microtwin stackings in the martensite. The complex microstructure with two martensite structures, multiple macro- and microtwinning and different out-of-phase type defects in $\mathrm{CuZr}$ is also discussed.
\end{abstract}

\section{INTRODUCTION}

The knowledge and understanding of the microstructure of a given phase or structure is essential for the proper interpretation of the general behaviour of the material, both from a fundamental as well as an engineering point of view. This microstructure can be related with the occurring phase transformations as well as the potential applications. The different operating modes of the electron microscope yield various types of information which will enable the investigator to obtain a general as well as detailed view of the material under study. In the case of martensitic transformations, the intrinsic nature of the transformation further places important restrictions on the possible microstructures. In turn, these microstructures can reveal typical characteristics of the transformation even when obtained from ex-situ experiments.

In the present contribution an overview will be presented of some of the recent results obtained in our current programs. These are concentrated on three different items. First our previous studies on precursor microstructures in $\mathrm{Ni}-\mathrm{Al}$ are extended to alloys of the Ni-Mn-Ti ternary configuration in which the same B2 to $\mathrm{L}_{0}$ martensitic transformation occurs but with different microtwin stacking sequences and different $\mathrm{M}_{\mathrm{s}}$ temperatures. In practise, a new micromodulation in distorted B2 precursor domains existing before the transformation towards a (22) structure could be recognised [1]. A second part will deal with the martensitic transformation in new types of $\mathrm{Ni}$-Al materials. We've investigated the microstructure of splat-cooled material and nanoscale thin films at different compositions comparable to those previously used in bulk studies. In the present text the general contexts and some typical microstructures are presented while more details are given in a separate contribution at this meeting [2]. The last part will consist of some examples of the particular microstructure in a different system, the $\mathrm{CuZr}$ alloy in which martensite structures similar to those in NiTi are found. A more extended description of the results of the latter study can be found in another contribution in these proceedings [3].

The experimental techniques used primarily involve conventional as well as high resolution transmission electron microscopy (CTEM and HRTEM) including in-situ heating and cooling investigations in the first mode as well as selected area electron diffraction (SAED). Some chemical analysis was also performed by standard energy dispersed X-ray analysis (EDX).

\section{PRECURSORS IN NI-MN-TI}

Previous HRTEM studies on the atomic and microstructure of the precursors in Ni-Al B2 suggested a possible correlation between the micromodulations inside the small distorted domains of a given shear variant and the stacking sequence of the ensuing martensite structure. It was shown that the existence of the modulation depends on the composition in a similar way as the appearance of a typical stacking sequence in the martensite. In fact, samples transforming thermoelastically into the (52) stacking of the $14 \mathrm{M}$ structure were always preceded by a micromodulation with an incommensurate wavelength of $1.3 \mathrm{~nm}$, i.e. between 6 
and $7(110)_{\mathrm{B} 2}$ planes [4]. At higher Ni content the martensite does not show a fixed stacking sequence while at the same time the micromodulation in the corresponding B2 precursor is lost $[5,6]$. Although a correlation between the precursor micromodulation and the martensite stacking could be inferred from these (and related [6]) experiments and although this possible correlation was confirmed by theoretical models [7], these observations remained limited to this single case study. Also alternative explanations for the existence of the particular stacking sequences were given [8].

The Ni-Mn(-Ti) systems exhibits the same martensitic transformation as the Ni-Al system. The austenite $\mathrm{B} 2$ phase transforms upon quenching into a close packed $\mathrm{L} 1_{0}$ type structure with multiple twinning on the close packed planes within a given plate $[9,10,11]$. For the ternary alloys, the Ti atoms are randomly distributed on the Mn lattice [11]. For the binary as well as for the ternary system slight changes in composition, i.e. e/a ratio, yield different stacking sequences for the martensite. In the binary system, however, the $\mathrm{M}_{\mathrm{S}}$ temperature is always far above room temperature which prohibits HRTEM studies of the precursors. This $\mathbf{M}_{\mathrm{S}}$ temperature can be decreased by adding $\mathrm{Ti}$ meanwhile changing the e/a ratio and the martensite stacking sequence. For one composition, Ni43.8 $\mathrm{Mn}_{42.4} \mathrm{Ti}_{13.8}$, it was possible to obtain an $\mathrm{M}_{5}$ temperature below but close to room temperature. As a result the precursors in the austenite could be imaged by HRTEM while at some specific locations or after electron irradiation of several minutes the martensitic structure was or became visible. In the present case the precursors revealed a micromodulation of $0.8 \mathrm{~nm}$ or $4(110)_{\mathrm{B} 2}$ planes as seen in fig. 1a and schematically represented in fig. $1 \mathrm{~b}$ while the martensite appeared with a (22) stacking seen in fig. 3b. Here the martensitic transformation was induced by irradiation of the sample with $400 \mathrm{keV}$ electrons for a few minutes, the growth of the plates being clear from the images in fig. $2 \mathrm{a}$ and $2 \mathrm{~b}$ taken after $1 \mathrm{~min}$. and $5 \mathrm{~min}$. irradiation, respectively. The Ni-Mn-Ti material used for the present study was prepared by P. Potapov of the Institute of Physical Metallurgy in Moscow.
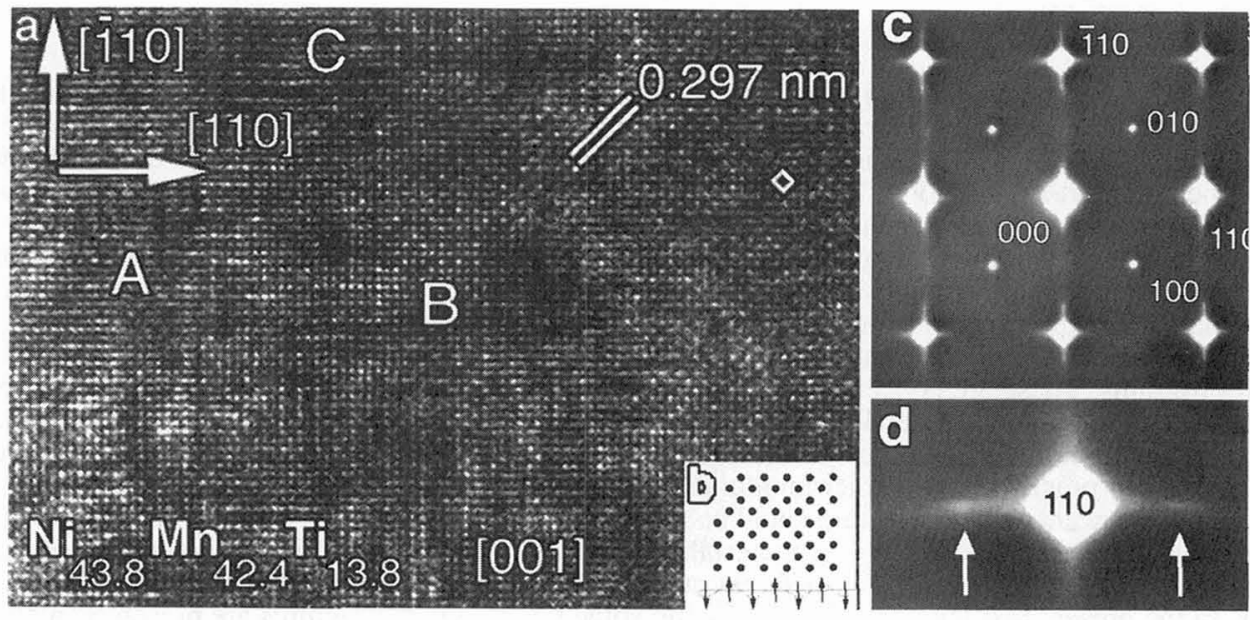

Figure 1. (a) [001] HRTEM image of the precursor distortions in the B2 in Ni43.8 Mn42.4 Ti13.8. (b) schematic of the periodic shuffle, (c) [001] SAED including diffuse $<110>$ streaks and (d) satellite in the $<110>$ diffuse streak at a 110 reflection.

The effect of the micromodulation in the distorted domains of the precursor is also visible in the SAED pattern. Indeed, in fig. 1d the satellite at the diffuse streak in the $\langle 110\rangle$ direction indicates the preference for a certain wavelength in the distortion. From measuring the actual ratio between the position of this satellite and that of the Bragg reflections the periodicity can be calculated. In the present case this would at first glance reveal a discrepancy between the real HRTEM image and the reciprocal SAED pattern. Indeed, the image shows a periodicity of 4 (110) planes whereas the satellite is located at a $1 / 5$ fraction of the 110 reciprocal distance. This difference, however, can fully be accounted for by the smallness of the distorted domains, approximately $2 \mathrm{~nm}$, from which it is concluded that the influence of the potential anti-phase nature of the modulations at the boundaries between two adjacent domains is included in the averaged positioning of the satellite. As a result the satellite is shifted to larger wavelengths. A simulation of the latter effect is shown in fig. 2 where the top row shows the models used as input for the power spectra in the bottom row. A modulation with a periodicity of 4 planes yields a satellite at a fraction of $1 / 4$ as long as the coherency 
length is long enough (left and right columns). When the domains are too small a fraction of $1 / 5$ is measured (central column).

The observation of the (2) $)$ stacking sequence for the martensite is in accordance with an earlier determination of this martensite structure by in-situ X-ray diffraction [11], which indicates that the electron irradiation only increases $M_{S}$ but does not affect the atomic structure.

The present result adds to the previous conclusion for $\mathrm{Ni}-\mathrm{Al}$ by again revealing a system in which the micromodulation of the B2 austenite already has the same distortion characteristics as those necessary to produce the correct martensite structure. Recently precursor effects were also observed in Ni-Mn-Al alloys preceding the transformation to the $14 \mathrm{M}$ structure but the exact atomic configuration still has to be elucidated [12].

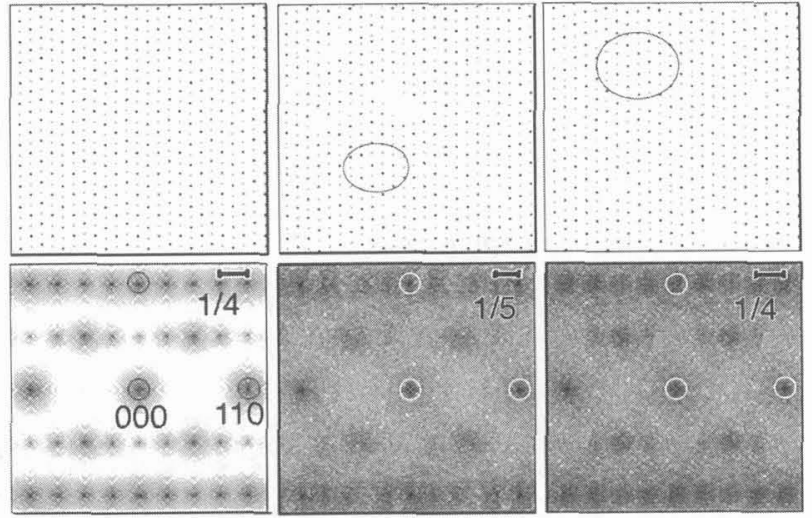

Figure 2. Simulation showing the effect of the domain size and the anti-phase nature of the domain boundaries on the position of the satellite in the diffuse intensity streak in the SAED pattern.
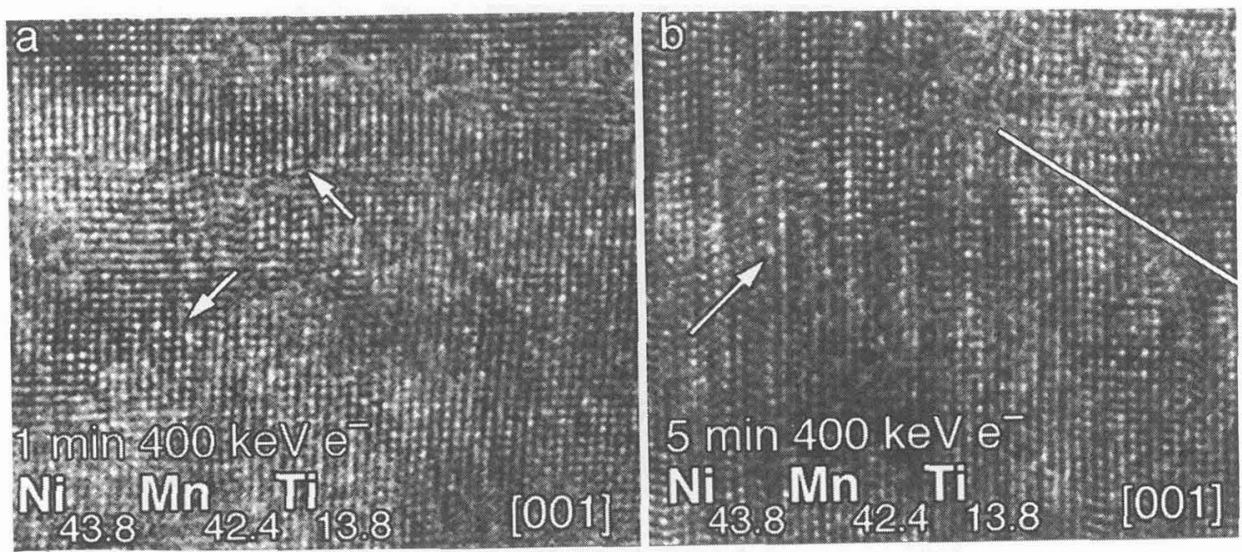

Figure 3. [001] HRTEM images of the (22) martensite stacking in Ni43.8 $\mathrm{Mn}_{42.4} \mathrm{Ti}_{13.8}$ after irradiation of the precursor austenite by $400 \mathrm{keV}$ electrons for (a) 1 minute and (b) 5 minutes.

\section{AUSTENITE AND MARTENSITE MICROSTRUCTURE IN SPLAT-COOLED NI-AL}

In the past many experiments have indicated that the defect configuration of an austenite phase can play an important role in the nucleation and growth processes of a martensitic transformation. The types of defects 
involved cover the whole range of zero- till three-dimensional ones, e.g. vacancies or interstitials ( 0-D), dislocations (1-D), grain boundaries (2-D) and precipitates (3-D). In the case of the martensitic transformation in Ni rich Ni-Al most studies until now only dealt with bulk material. Well homogenised bulk samples have relatively large B2 austenite grains, sometimes with dimensions up to a few $\mathrm{mm}$, with few dislocations. The most prominent defects in this system are vacancies and the excess $\mathrm{Ni}$ atoms, i.e. with respect to the 1-1 ratio of the B2 structure, which are randomly distributed on the Al sublattice $[13,14]$. Configurations of such antisites are potential candidates of tetragonal strain centres forming nucleation centres for the inhomogeneously strained domains (ISD's) recognised in the precursor state (cf. chapter 2) [7]. Also quenched-in vacancies can have an important effect on the martensitic or related transformations such as the formation of the metastable $\mathrm{Ni}_{2} \mathrm{Al}[15,16,17]$ and stable $\mathrm{Ni}_{5} \mathrm{Al}_{3}$ phases $[18,19]$.

In order to enlarge the available information on the $\mathrm{Ni}-\mathrm{Al}$ system new preparation techniques have been applied to this material. Samples with a Ni content of 62.5 and 65 at.\% Ni were splat-cooled from the melt between two flat $\mathrm{Cu}$ discs at a rate of $510^{5} \mathrm{~K} / \mathrm{s}$. This was performed by D. Holland-Moritz from the Institut für Raumsimulation, Köln, Germany. The resulting discs have a diameter of approximately $20 \mathrm{~mm}$ and a thickness of about $200 \mu \mathrm{m}$. From these $3 \mathrm{~mm}$ EM samples were prepared by the conventional electropolishing technique for $\mathrm{Ni}-\mathrm{Al}$ alloys [4].

Samples containing 62.5 at.\% Ni primarily reveal the B2 phase in small grains of a few $\mu \mathrm{m}$ in diameter. Adjacent grains are often seen to have a very similar orientation for their normal as seen from the overview and SAED patterns in fig. 4. The grains 1 and 2 or 3 are observed along or close to the [001] zone, respectively, but show a relative rotation of approximately $45^{\circ}$ around this zone axis. Some of these grains are partially transformed into the microtwinned $L 1_{0}$ martensite. Such sites are indicated by $M$ on fig. 4 . Occasionally this martensite reveals a stacking close to that of the 14M structure [20]. An example of the latter as well as some more detail on the occurring interfaces is presented in ref. [2].

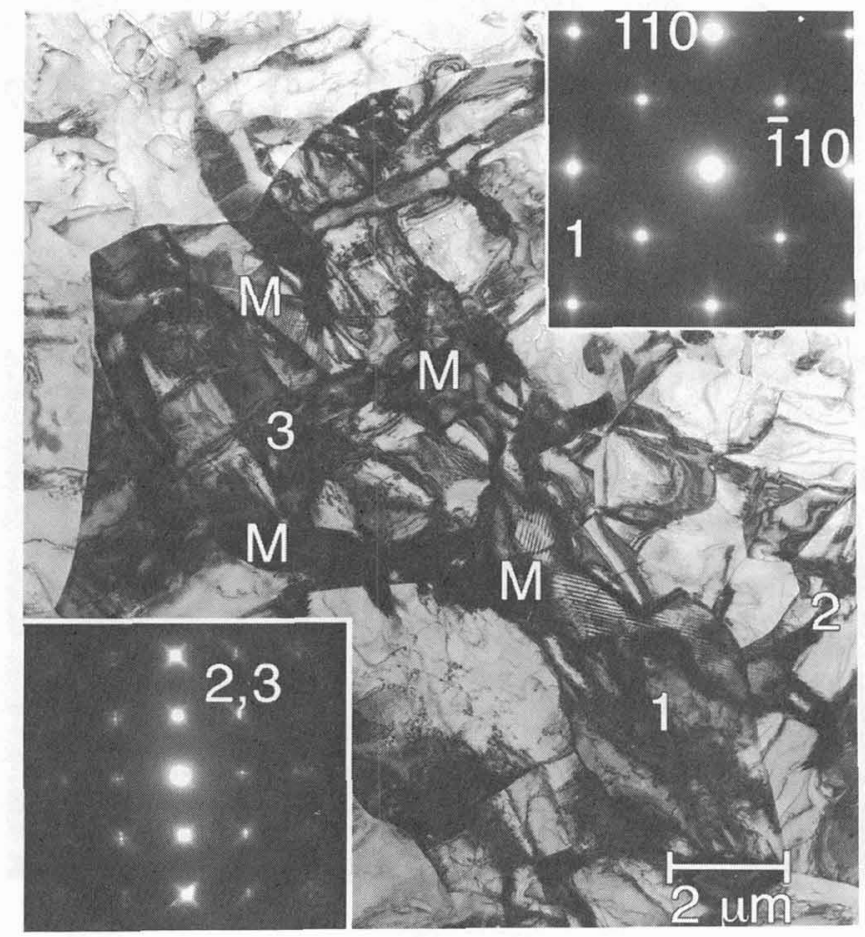

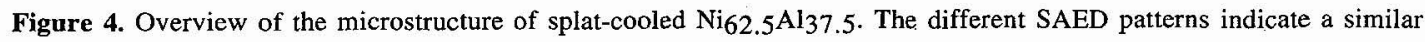
orientation of the normals of adjacent grains 1 and 2 or 3 .

The [001] zone pattern in fig. 4 also shows that these B2 grains are in the precursor state which is clear from the typical $\langle 110\rangle$ diffuse intensity streaks in reciprocal space. These streaks, however, do not show any intensity enhancements or satellites which can be correlated with the fact that no micromodulations are seen under HRTEM conditions [4]. This is shown in fig. 1 of ref. [2]. 
In splat-cooled $\mathrm{Ni}-\mathrm{Al}$ with 65 at.\% $\mathrm{Ni}$ the material is nearly completely transformed into martensite. Some grains are completely transformed into a single microtwinned martensite plate while occasionally the impression of a self-accommodating group is found. Examples of both cases are found in figs. 5a and 5b, respectively. As seen from these CTEM images the microtwinning yields relatively broad twin variants although finer ones are also observed.
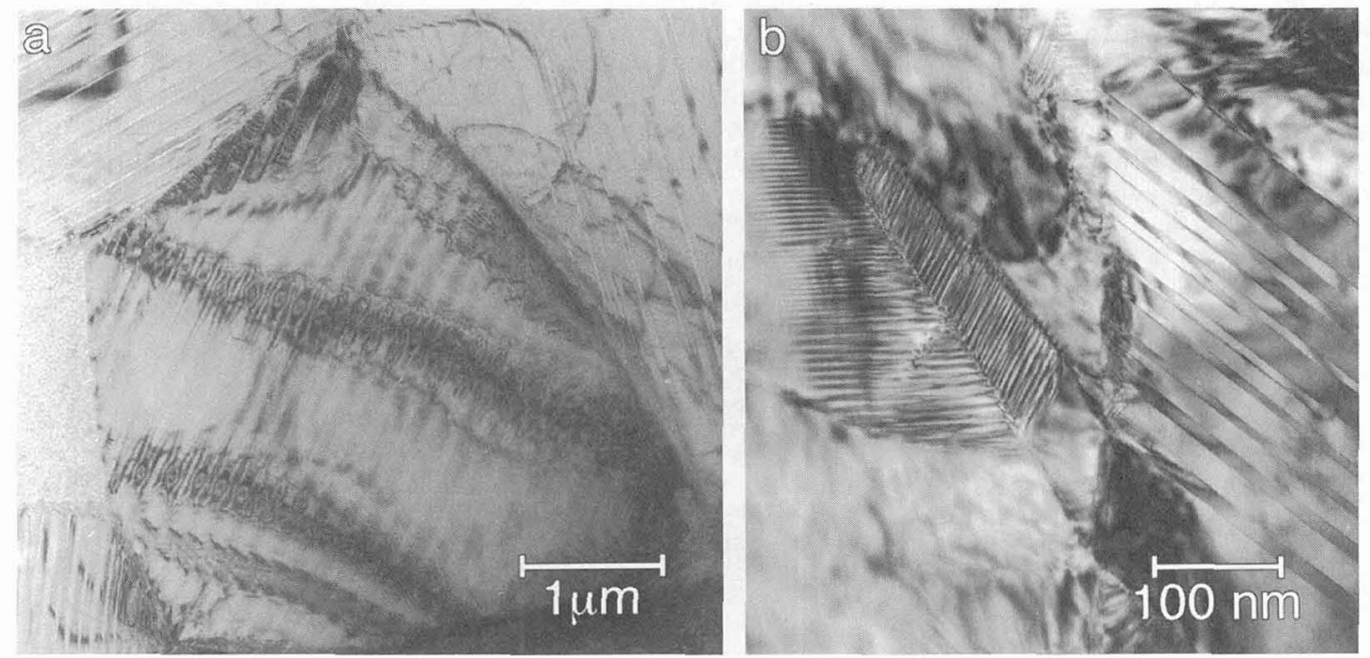

Figure 5. Overview of the microstructure of splat-cooled $\mathrm{Ni}_{65} \mathrm{Al}_{35}$ showing (a) single grain martensite plates and (b) a selfaccommodating group of plates.

An interesting observation is found in some grains where the microtwin stacking is far from regular when considering the entire plate. This is seen in fig. $6 \mathrm{a}$ where in some parts a typical microtwin sequence is found while at regular intervals a very wide variant exists. In most cases these strong deviations can be correlated with distinct steps or facets at the interface. To what extend the shape of the original interface between two adjacent austenite grains plays a role in the formation of this configuration is still unclear. In fig. $6 \mathrm{~b}$ a stairway shape is formed at an interface where two martensite plates meet within a given B2 grain and with their microtwin planes nearly perpendicular. Again different stacking sequences can be correlated with the interface structure. HRTEM images of some other grain boundaries also including a 14M-like stacking can be found in ref. [2].
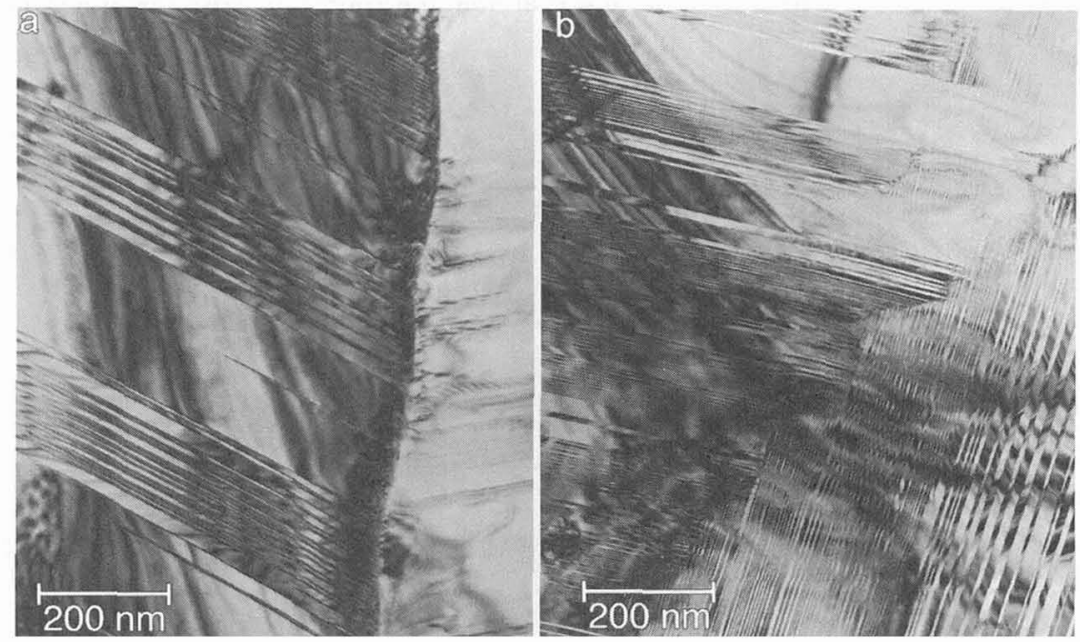

Figure 6. (a) Effect of a B2 grain boundary on the martensite microtwin stacking sequence and (b) a stairway interface between two martensite plates formed inside a single $\mathrm{B} 2$ grain, both in splat-cooled $\mathrm{Ni}_{65} \mathrm{Al} 35$. 


\section{NANOSCALE NI-AL THIN FILMS}

Different types of evaporated $\mathrm{Ni}-\mathrm{Al}$ films of nanosize thickness were prepared on $\mathrm{NaCl}$ or $\mathrm{KCl}(001)$ substrates. The original idea behind this study was to investigate the martensitic transformation under morphological conditions which are well defined, i.e. much better than in the case of polycrystalline bulk material. Depending on the actual goal in mind, the most ideal configuration could be a single crystal thin film of austenite with a simple normal direction, e.g. [001], or a polygrain film with variable grain size. In the latter case different degrees of epitaxy could be envisaged. When the film thickness does not exceed hundred nanometer such samples are directly suitable for CTEM, SAED and HRTEM studies without the need for further thinning. Here two typical results are presented while more detail can be found in ref. [2]. The present thin films were prepared by M. Yandouzi and L. Toth at the Institute for Thin Film Research in Budapest, Hungary.

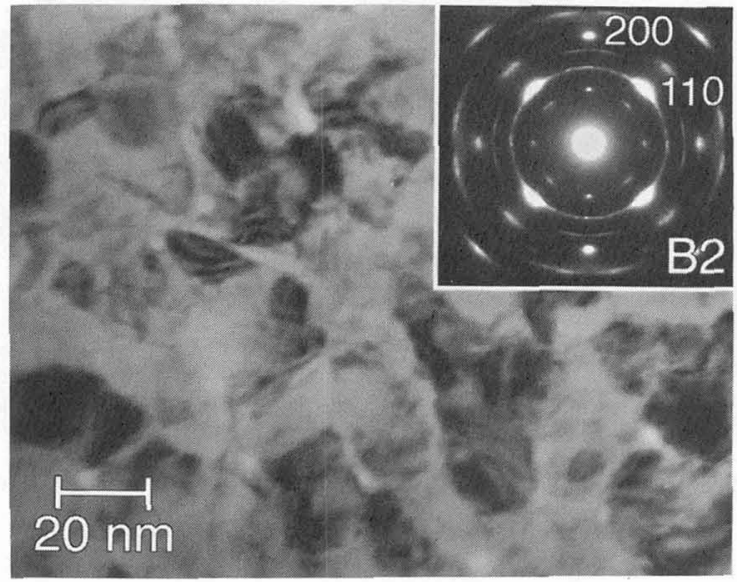

Figure 7. SAED and microstructure of a $\mathrm{B} 2 \mathrm{Ni} 62.5 \mathrm{Al} 37.5$ thin film evaporated onto (001) $\mathrm{NaCl}$ at $300{ }^{\circ} \mathrm{C}$.

Evaporating a $\mathrm{Ni}_{62.5} \mathrm{Al}_{37.5}$ alloy for $100 \mathrm{~s}$ onto an ex-situ cleaved $\mathrm{NaCl}(001)$ surface in a vacuum of $1.510^{-5}$ mbar yielded a nearly contiguous monophase crystalline film of B2 grains of a few nm in diameter as seen in fig. 7. When the substrate is heated up to $300^{\circ} \mathrm{C}$ during deposition some preferential epitaxy appears. In the inset of fig. 7 an SAED pattern of such a film is shown. The most common epitaxial relation is $(001) / /(001),[100] / /[100]$ as seen from the most intense reflections in the 110 (second) and 200 (fourth) rings. Still, some weaker reflections also reveal the existence of a $(001) / /(001),[100] / /[110]$ epitaxy, i.e. with the same contact plane but with a rotation of $45^{\circ}$ around the zone axis. HRTEM images of such films are presented in ref. [2].

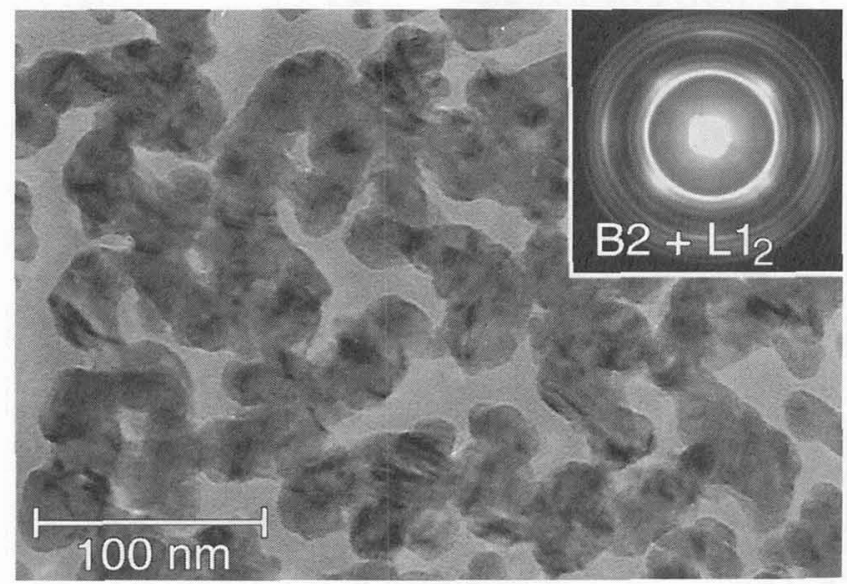

Figure 8. SAED and microstructure of a $\mathrm{Ni}_{62.5} \mathrm{Al} 37.5$ thin film evaporated onto (001) $\mathrm{KCl}$ at $450{ }^{\circ} \mathrm{C}$ revealing (a) $\mathrm{B} 2$ as well as $\mathrm{Ll}_{2}$ rings and (b) a less contiguous film. 
When using a $\mathrm{Ni}_{65} \mathrm{Al}_{35}$ alloy as evaporation source under similar conditions (i.e., on $\mathrm{KCl}$ at $360^{\circ} \mathrm{C}$ ) virtually the same results are obtained but the preferred epitaxy is less well established and the grain size is some $20 \%$ smaller than in the previous case. On the other hand, when the substrates are held at $450^{\circ} \mathrm{C}_{,} \mathrm{L1}_{2}$ rings are clearly observed as seen in the inset of fig. 8. Such films are also usually less contiguous as is clear from the microstructure in fig. 8.

When the appearance of a monophase B2 film at these compositions and lower temperatures is compared with the phase diagram of the bulk material it is seen that in the latter case one would expect a decomposition with the $\mathrm{L}_{2}$ or $\mathrm{Ni}_{5} \mathrm{Al}_{3}$ phases. Rings from these phases, however, are clearly missing from the diffraction pattern in fig. 7. Averaged as well as microprobe EDX analysis revealed a slightly Ni enriched composition with respect to the evaporation source. Composition differences between the evaporation source and the final film are a well known fact in thin film experiments. The high Ni content of single B2 grains as measured by microprobe EDX confirms that no decomposition has taken place and that the formation of the B2 grains is possible aided by the epitaxial relations with the substrate. When the substrate is held at higher temperatures the influence of the epitaxy is decreased and the expected decomposition takes place.

When the monophase samples are cooled to $-173^{\circ} \mathrm{C}$ no martensitic transformation is observed. In view of the measured compositions which have an $\mathrm{M}_{\mathrm{S}}$ around $400^{\circ} \mathrm{C}(65$ at. $\% \mathrm{Ni})$ or between 0 and $-100^{\circ} \mathrm{C}$ $(62.5$ at.\% Ni) for bulk material, the nanoscale features of the film presumable inhibit the martensitic transformation. Indeed, recent results in other materials also indicate that the austenite can strongly be stabilised by the size of the grains or the introduction of defects such as dislocations, grain boundaries and point defects $[21,22]$. The overwhelming number of grain boundaries is obvious in the present case while the relatively low temperature of the substrate can be assumed not to provide enough diffusion for proper ordering kinetics and thus leaving the austenite with a high density of vacancies and antisite defects.

\section{MARTENSITE IN CUZR}

Recently a new group of alloys exhibiting martensitic and shape memory characteristics has caught the attention of several researchers [23]. Among these the binary $\mathrm{Cu}-\mathrm{Zr}$ system appears to be a good representative [24]. In practise it turns out that the martensitic transformation is similar to that in $\mathrm{Ni}-\mathrm{Ti}$, which is not surprising considering the elements involved [25,26,27]. Macroscopic experiments, however, indicated a rather complex behaviour of the material during the transition before and after cycling or by the addition of ternary elements $[28,29]$. In order to help to clarify some of the questions in this respect an extensive investigation of the atomic and microstructure of the CuZr martensite was started. In a first step the $X$-ray data was confronted with lattice parameters and space group symmetry obtained from detailed SAED tilting experiments which lead to the refinement of the atomic positions in the product structures [27]. In most samples two different martensitic structures were found, the smallest one being of the B19'. type while the largest one could be considered as a superstructure of this B19' cell [27]. Because of the existence of these two related structures complicated microstructural arrangements appear in the martensite plates. In this part we will elaborate on these configurations while more detail on the atomic scale will be presented on the basis of more HRTEM images in another contribution at this meeting [3]. The crystallographic indices used further on will refer to the largest unit cell. Some of the samples used in the present work were prepared by G.S. Firstov of the Institute of Metal Physics in Kiev, Ukraine.
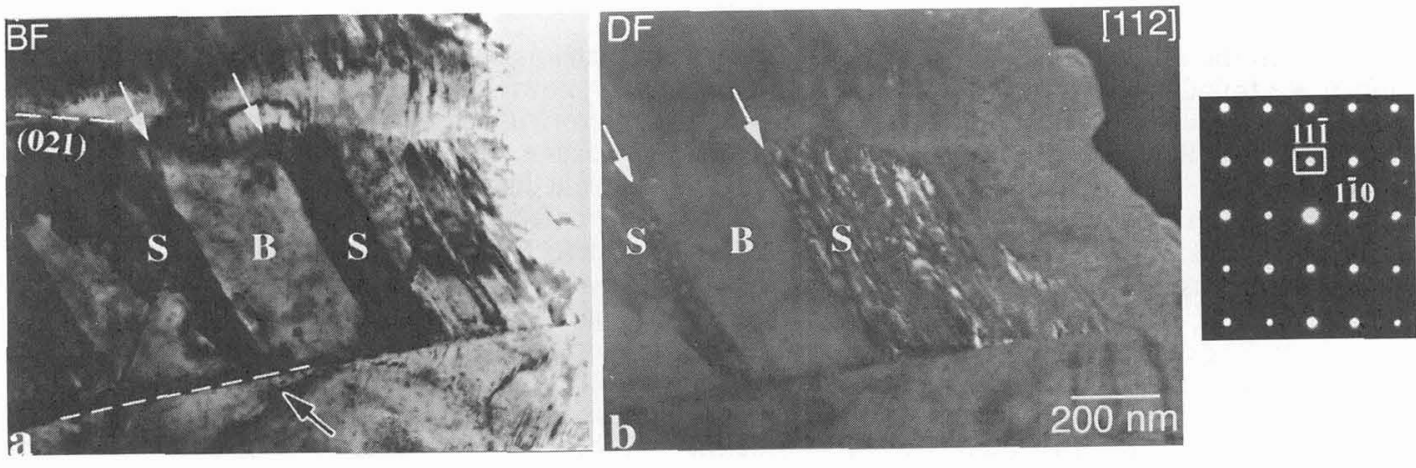

Figure 9. (a) BF and (b) DF image of a martensite plate including regions of different structures and microtwins in CuZr. 
In fig. 9 a bright and dark filed (BF and DF) image of a typical martensite plate in CuZr is shown together with the respective SAED pattern. Because of the different symmetry and size of the unit cells of the two appearing structures some satellites appear in this particular [112] orientation. The extra rows are indicated by arrows in fig. 9c. The DF image shows that both structures exist in one martensite plate while their locations can roughly be correlated with microtwin traces visible in the BF image. From this and other images it is found that the supercell is often twinned on (001) planes while no microtwins are found in the region of the basic cell [30]. The boundary between these two regions is usually parallel with the microtwin planes although deviations from this are also observed (black-white arrow in fig. 9a). One of the martensite plate boundaries is parallel with the $(021)$ plane of the supercell which originates from the $(010)$ plane in the B2 austenite. This plane is often observed as a macrotwin interface between two martensite plates [3]. From the scale bar it can be concluded that the present martensite plates are very small, the one shown in fig. 9 being selected for its large dimensions.

Due to the small size of the plates, regular arrangements of accommodating plates are difficult to visualise. One such an example is shown in fig. $10 \mathrm{a}$ where the $(021)$ boundaries are again recognised and the midrib of the spear is a (332) plane of the superstructure martensite. Both plates are observed along a [110] zone which yields the SAED pattern of fig. 10b. The angle between the microtwins on both sides of the midrib is $49^{\circ}$ which can also be measured from the SAED pattern. This data corresponds well with that for the B19' structure in NiTi which again indicates that both transformations are isostructural [31].
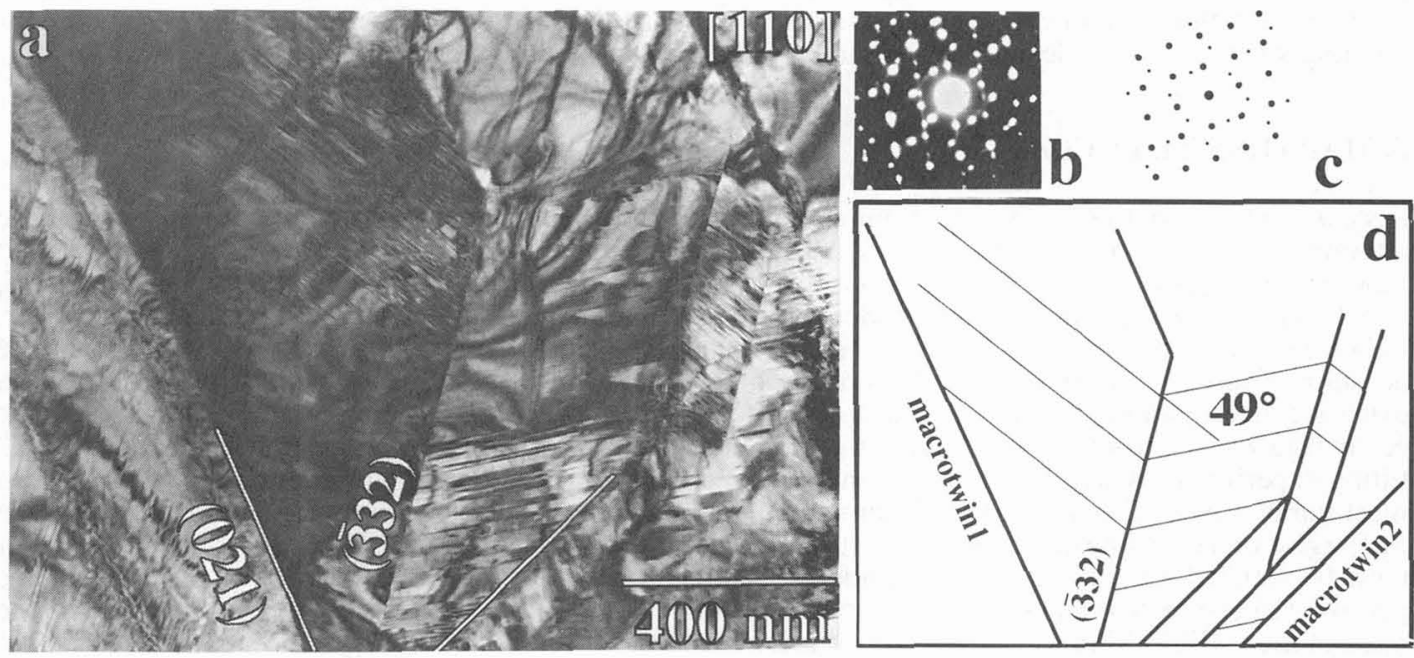

Figure 10. (a) BF image and (b), (c) SAED pattern of a martensite spear configuration with the respective measurements indicated on the schematic in (d).

When the internal microstructure of the martensite plates is studied in more detail some singular defects are found crossing the microtwins. A first example is shown in the CTEM image of fig. 11 where sharp lines indicated by white arrows are crossing the microtwins at an angle of approximately $21^{\circ}$. Although these lines look straight at this magnification, a closer examination including HRTEM images reveals that they contain many atomic steps and that they have a displacement vector $\vec{R}=1 / 4[1 \overline{1} 0]$. In a [11i ] orientation these lines are seen to be the traces of planar defects observed edge-on but without a well defined habit plane due to the atomic steps. In ref. [3] these defects are called type A. They also continue into those regions where no microtwins are observed. Also they do not seem to influence the microtwins, i.e. the latter give the impression to continue over the defect without changing their microtwin plane.

In fig. $12 \mathrm{a}$ a very small martensite plate is seen to stop inside a larger one at an irregular defect observed edge-on in the present [110] orientation. This defect is not confined to a given crystallographic plane nor are those seen in the image of fig. $12 \mathrm{~b}$. Both these images are examples of defects of type B studied in detail in ref. [3]. From fig. $12 \mathrm{~b}$ it is clear that these defects have a strong influence on the microtwins: indeed, most microtwins end at the defect which results in a different microtwin arrangement on both sides of the defect. At locations where the microtwin variant is the same on both sides of the defect, HRTEM images reveal a displacement vector $\bar{R}=1 / 4[102]$ [3]. 


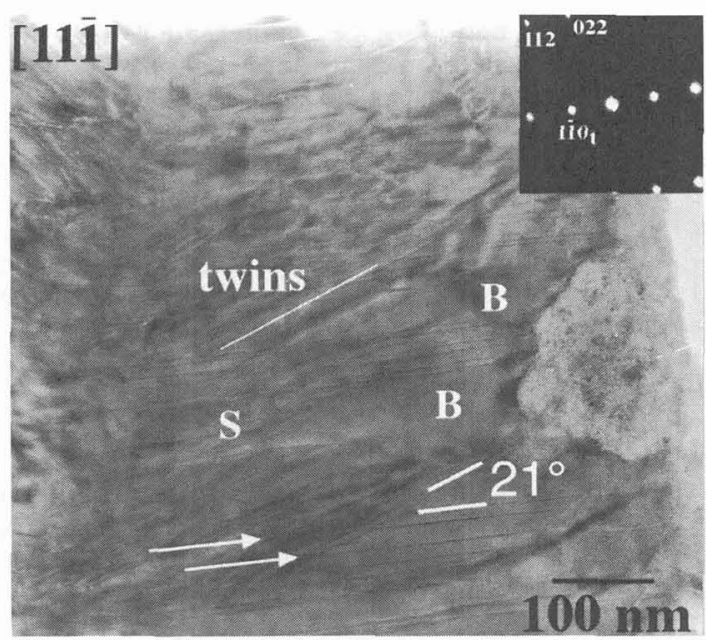

Figure 11. CTEM image of type A defects in CuZr martensite crossing the microtwin planes.
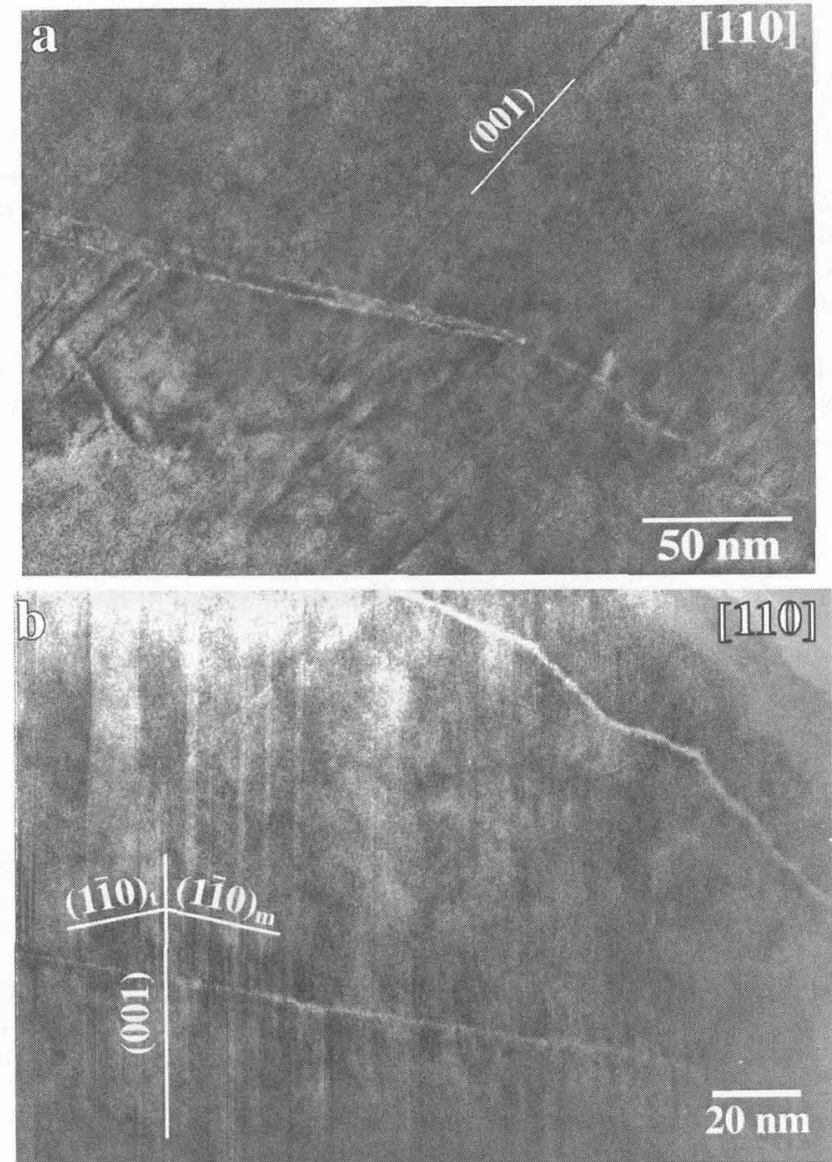

Figure 12. Low magnification HRTEM images of type B defects in CuZr martensite. In (a) the defect is attached to the tip of a martensite plate while in (b) the different microtwin arrangement on either side is obvious. 
From the details of the atomic resolution images as presented in ref. [3], it is concluded that both defects, i.e. type A as well as B, are inherited from anti-phase boundaries in the austenite. Because of the combination of a shear and shuffle mechanism necessary to produce both martensitic phases, two different defects can be formed in the martensite starting from the only anti-phase boundary possible in the austenite, i.e. with a displacement vector of $\bar{R}=1 / 2[111]$. The main difference between the two types is that for type $A$ the sublattice containing the shuffling atoms remains fixed over the defect while for type $B$ this lattice changes to the alternative possibility, i.e. simply a shift of origin. As these defects appear randomly inside the martensite plates it can be inferred that they are simply transformed together with the matrix. Those which accidentally make a larger angle to the microtwin planes have a stronger interaction with the latter which results in a change of shuffle lattice and microtwin plane.

\section{Acknowledgements}

I wish to thank Ludo Rossou for help with the thin film preparations and Dr. V. Teodorescu from the Institute of Atomic Physics, Bucharest-Magurele, Romania, for many stimulating discussions on the thin films. Part of the electron microscopy on the Ni-Al thin films was performed by M. Yandouzi while most of the work on the $\mathrm{Cu}-\mathrm{Zr}$ was done by J.W. Seo who was employed under IUAP contract nb. 46.

\section{References}

[1] Schryvers D., Lahjouji D.E., Slootmaekers B. and Potapov P.L., Scripta metall. mater. 35 (1996) 1235-1241.

[2] Schryvers D., Yandouzi M. and Toth L., these proceedings (1997).

[3] Seo J.-W. and Schryvers D., these proceedings (1997).

[4] Schryvers D. and Tanner L.E., Ultramicroscopy 32 (1990) 241-254.

[5] Schryvers D., habilitation, V.U.B. (1991).

[6] Schryvers D., "Experimental studies on precursor phenomena in displacive phase transformations", proc. First Conference on Alloys, Athens, Greece, 1996, (A. Gonis and P. Turchi, Eds.), (to be publ.).

[7] Gooding R.J. and Krumhansl J.A., Phys. Rev. B 39 (1989) 1535-1540.

[8]. Khatchaturyan A., Shapiro S.M. and Semenovskaya S., Phys. Rev. B 43 (1991) 10832-10843.

[9] Baele I., Van Tendeloo G. and Amelinckx S., Acta metall. 35 (1987) 401-412.

[10] Potapov P.L., Scripta Metall. Mater. 31 (1994) 1243.

[11] Udovenko V.A., Potapov P.L., Polyakova N.A. and Prieb V., Z. Metallkd. 86 (1995) 345-352.

[12] Kainuma R., Nakano H. and Ishida K., Metall. Mater. Trans. A 27 (1996) 4153-4162.

[13] Georgopoulos P. and Cohen J.B., Acta metall. 29 (1981) 1535-1551.

[14] Bradley A.J. and Taylor A., Proc. Roy. Soc. Lon. A 159 (1937) 56-72.

[15] Schryvers D., Toth L., Van Humbeeck J. and Beyer J., "Ni2 Al versus $\mathrm{Ni}_{5} \mathrm{Al}_{3}$ ordering in $\mathrm{Ni}_{65} \mathrm{Al}_{35}$ austenite and martensite", proc. ICOMAT, Lausanne, Switzerland, 1995, (R. Gotthardt and J. Van Humbeeck, Eds.), pp. 1029-1033.

[16] Muto S., Merk N., Schryvers D. and Tanner L.E., Phil. Mag. B 67 (1993) 673-689.

[17] Muto S., Schryvers D., Merk N. and Tanner L.E., Acta metall. mater. 41 (1993) 2377-2383.

[18] Schryvers D., Ma Y., Toth L. and Tanner L.E., Acta metall. mater. 43 (1995) 4045-4056.

[19] Schryvers D., Ma Y., Toth L. and Tanner L.E., Acta metall. mater. 43 (1995) 4057-4065.

[20] Martynov V.V., Enami K., Khandros L.G., Tkachenko A.V. and Nenno S., Scripta Metall. 17 (1983) 1167-1173.

[21] Kajiwara S., Metall. Trans. A 17 (1996) 1693-1702.

[22] Murakami Y., Nakajima Y. and Otsuka K., Scripta metall. mater. 34 (1996) 955-962.

[23] Koval Y.N., Firstov G.S., Van Humbeeck J., Delaey L. and Jang W.Y., "B2 intermetallic compounds of Zr. New class of the SMA", proc. ICOMAT, Lausanne, Switzerland, 1995, (R. Gotthardt and J. Van Humbeeck, Eds.), pp. 1103-1108.

[24] Koval Y.N., Firstov G.S. and Kotko A.V., Scripta metall. mater. 27 (1992) 161 1-1616.

[25] Bührer W., Gotthardt R., Kulik A., Mercier O. and Staub F., J. Phys. F 13 (1983) L77.

[26] Kudoh Y., Tokonami M., Miyazaki S. and Otsuka K., Acta metall. 33 (1985) 2049.

[27] Schryvers D., Firstov G.S., Seo J.-W., Van Humbeeck J. and Koval Y.N., Scripta metall. mater. (1997) (to be published).

[28] Koval Y.N., Firstov G.S., Delaey L. and Van Humbeeck J., Scripta metall. mater. 31 (1994) 799-802.

[29] Jang W.Y., Van Humbeeck J., Delaey L., Koval Y.N. and Firstov G.S., "The influence of Ti and Ni additions and thermal cycling on the Martensitic transformation in $\mathrm{Cu}-\mathrm{Zr}$ alloys", proc. Advanced Materials '93, 1993, (K. Otsuka and e. al., Eds.), pp. 1009-1012.

[30] Seo J.W. and Schryvers D. (to be published).

[31] Mulder J.H., Ph.D. thesis, University of Twente (1995). 